\title{
Toward a Constructivist Epistemology of Thought Experiments in Science
}

\author{
Kristian Camilleri \\ School of Historical and Philosophical Studies \\ University of Melbourne \\ Australia 3010 \\ kcam@unimelb.edu.au \\ $+61383447573$
}

\begin{abstract}
This paper presents a critical analysis of Tamar Szabó Gendler's view of thought experiments, with the aim of developing further a constructivist epistemology of thought experiments in science. While the execution of a thought experiment cannot be reduced to standard forms of inductive and deductive inference, in the process of working though a thought experiment, a logical argument does emerge and take shape. Taking Gendler's work as a point of departure, I argue that performing a thought experiment involves a process of self-interrogation, in which we are compelled to reflect on our pre-existing knowledge of the world. In doing so, we are forced to make judgments about what assumptions we see as relevant and how they apply to an imaginary scenario. This brings to light the extent to which certain forms of skill, beyond the ability to make valid logical inferences, are necessary to execute a thought experiment well.
\end{abstract}

\section{Keywords}

Thought experiments

Gendler

Cognitive skill

Constructivism

Science

Imaginary scenarios 


\section{Towards a Constructivist Epistemology of Thought Experiments in Science}

\section{Introduction}

How is it that by contemplating what would happen in an imaginary scenario, it is possible to acquire new knowledge or understanding of the physical world? This question, posed by Kuhn some fifty years ago, has been the subject of much recent debate from philosophers of science (Kuhn 1977). Over the last two decades a range of different philosophical positions has emerged, which attempt to develop an epistemology of thought experiments in the natural sciences. Tamar Szabó Gendler's constructivist epistemology, first articulated in her classic 1998 article on Galileo's falling bodies thought experiment, is one of the more intriguing and original perspectives to have emerged from the recent debates (Gendler 1998). Gendler rejects the widely held view that the 'demonstrative force' of a thought experiment can be explained by the fact that it can be reconstructed as a logical argument. Instead, she agues that carrying out a thought experiment involves a form of reasoning that cannot be captured in standard forms of logical argument, and which necessarily involves the contemplation of an imaginary scenario (Gendler 2000, 2002, 2004).

Gendler's view offers many important and valuable insights, however it suffers from a number of difficulties that require more careful consideration. This paper subjects Gendler's work to a critically analysis, with the aim of developing further a constructivist epistemology of thought experiments. According to the view I develop here, and in line with Gendler's basic contention, the execution of a thought experiment cannot be reduced to standard forms of inductive and deductive inference. However, this is not to deny that in the process of working though a thought experiment, a logical argument does emerge and take shape. Here it is decisive to realize that the premises (both explicit and implicit) of the reconstructed logical argument, no less than the conclusion, may emerge as a result of contemplating an imaginary scenario. Taking Gendler's work as a point of departure, but also drawing on the work of other philosophers like David Gooding, I offer some conclusions about the kind of reasoning employed in 'working through' a thought experiment, and how this differs from standard forms of inductive and deductive reasoning. 
The first half of the paper presents a critical exegesis of Gendler's view, with a particular focus on her analysis of Galileo's famous two bodies thought experiment. Here I begin by highlighting an important difference between the views of Gendler and John Norton. Whereas Norton is primarily interested in the logical form of the argument, by means of which we infer the conclusion of a thought experiment, Gendler focuses on the way we form beliefs, which serve as the premises of the argument. Understanding this difference turns out to be crucial in pinpointing the real source of their disagreement, and serves to highlight the different senses in which Gendler and Norton understand the 'justificatory force' of a thought experiment. Gendler's analysis of Galileo's famous two bodies thought experiment is then summarized. For Gendler, this example serves to illustrate the way in which the contemplation of an imaginary scenario allows us to tap into previously unarticulated tacit knowledge about the world. Yet, as I will show, this conclusion may be criticized on two counts: first, it rests on the problematic view that tacit beliefs may count as a reliable source of knowledge, and secondly, it overlooks the fact that many thought experiments rely on theoretical and well-articulated forms of knowledge. This is particularly evident in many classic thought experiments in modern physics.

But if a thought experiment is not an (inductive or deductive) argument, what exactly is it? In the second half of the paper, I attempt to address this question by developing further the constructivist viewpoint. As Gendler rightly points out, the execution of a thought experiment involves confronting a series of questions of the type: "What would I say/judge/expect were I to encounter circumstances XYZ?" (Gendler 1998, p. 414). However, whereas Gendler argues that in working through a thought experiment, we are guided by tacit beliefs about the world, I do not see this as a characteristic feature of all thought experiments. Different thinkers often make different assumptions and draw different conclusions from consideration of the same scenario. Here I argue that it is the questions we are forced to confront, not the answers, that prove to be of fundamental importance in understanding how thought experiments work. To this extent, I argue that thought experiments involve a process of selfinterrogation, which compel us to reflect on our pre-existing knowledge of the world. In executing a thought experiment we are forced to make judgments on what basic assumptions we take to be relevant to the phenomenon under consideration. In the process of working through a thought experiment these assumptions may be clarified, revised and refined. Indeed the very questions we pose in contemplating an imaginary scenario may even be reformulated.

In the final section I develop the constructivist view further by turning my attention to the kind of know-how or skill that is involved in performing a thought experiment. Here we can 
provide an answer to the question of how we are to distinguish 'good' from 'bad' thought experiments, quite apart from our ability to execute a logical argument. One skill, which is indispensible for the execution of a thought experiment, is the ability to recognize the relevance of some piece of (empirical) knowledge to the situation at hand. While it is often taken for granted that this is trivial, in many cases it is not. The process of working through a thought experiment depends not only on having previously acquired knowledge, but also 'seeing' its relevance for the phenomenon under consideration. The Bohr-Einstein debate at the 1930 Solvay conference proves to be a case in point. Furthermore, even when we recognize the relevance of our background knowledge, it is not always immediately obvious how to apply that knowledge to the imaginary scenario. In such cases, it becomes apparent that judgments about what is relevant and how to apply our knowledge play an indispensible role in the execution of a thought experiment. To this end, while thought experiments may take a logical form once we have worked through them, they cannot be understood simply as constituting an exercise in inductive or deductive inference.

\section{Norton and Gendler: Logical Arguments or Guided Contemplations?}

In order to begin the analysis of Gendler's account, it is first useful to contrast it with John Norton's view. Norton has argued that the principal conclusion drawn from a thought experiment is actually arrived at by means of standard forms of inductive or deductive inference, though typically they will include hidden assumptions and suppressed premises (Norton 1996, 2004a, 2004b). According to Norton, the fact that we can reliably judge whether the conclusion of a thought experiment is justified or not gives us good reason to think they are "governed by a generalized logic" (Norton 2004b, p. 54). On this account, we may simply replace a thought experiment with a logical argument, because a thought experiment is nothing but a disguised argument. This view rests on two different theses, which we may term the reconstruction thesis and the execution thesis:

Reconstruction Thesis: All thought experiments can be reconstructed as arguments based on tacit or explicit assumptions. Belief in the outcome-conclusion of the thought experiment is justified only insofar as the reconstructed argument can justify the conclusion. 
Execution Thesis: The actual conduct of a thought experiment consists of the execution of an argument, although this may not be obvious, since the argument may appear only in abbreviated form and with suppressed premises (Norton 2004b, p. 50). ${ }^{1}$

According to the reconstruction thesis, because it is possible to reconstruct a thought experiment as an argument, we are able to judge whether its conclusion is justified. The execution thesis, however, goes further - it states that not only can thought experiments be reconstructed as arguments, but deep down they are arguments. In support of this view, Norton appeals to a "long tradition in informal logic", according to which, the kind of reasoning we employ in working through hypothetical scenarios typically involves a disguised form of standard argumentation. Moreover, he claims it would be an extraordinary coincidence if the process of reasoning employed in carrying out a thought experiment were fundamentally different from the logical form of the reconstructed argument. If thought experiments were anything other than disguised forms of inductive or deductive inference, Norton contends, it would be impossible to submit them to logical analysis.

While the reconstruction thesis seems uncontroversial, the execution thesis has met with criticism from several authors. (Arthur 1999; Bishop 1998; Bokulich 2001; Gooding 1994) David Gooding puts the point succinctly: "Even granting that any TE [thought experiment] can be reconstructed as a deductive argument with empirical premises, it does not follow that TEs are just deductive arguments, nor does this explain how they work" (Gooding 1994, p. 1035). In Gooding's view, a thought experiment should be understood first and foremost as "a process to be worked through, rather than a logical structure", and to this extent the logical reconstruction "is possible only when the working-through has been successfully completed" (Gooding 1993, p. 283). Gendler adopts a similar view in drawing a contrast between the way in which we "draw a conclusion on the basis of a thought experiment" and the "process of inductive or deductive reasoning" by which we draw a conclusion on the basis of a nonthought experiment argument" (Gendler 1998, p. 399). As Gendler puts it, Norton's view "requires accepting that something that feels like the contemplation of an imaginary scenario is actually the execution of an argument" (Gendler 2004, pp. 1153-4).

In Gendler's view, not only does the logical reconstruction of a thought experiment differ, in some important epistemological sense, from the original execution of the thought experiment, but it also fails to capture the original justificatory force of a thought experiment (Gendler

\footnotetext{
${ }^{1}$ This kind of logical reconstruction has been carried out on many thought experiments including Galileo famous falling bodies thought experiment (Schrenk, 2004).
} 
1998 p. 401). ${ }^{2}$ Reconstructing a thought experiment as a deductive argument may of course be possible and even valuable, but this does not mean that it is "epistemically equivalent to the original thought experiment" (Arthur 1999, p. 219). This is because, for Gendler, "the justificatory force of the thought experiment actually comes from the fact that it calls upon the reader to perform ... an experiment-in-thought", not from the fact that it can be reconstructed as a logical argument (Gendler 1998, pp. 413-4). To this extent, the process of working through a thought experiment "may, in certain contexts, be sufficiently reliable to count as a source of justification" (Gendler 2004, p. 1154). The beliefs we acquire in the course of executing a thought experiment are justified in a quite different fashion than is the case in standard forms of deductive and inductive inference.

Here it seems that Norton and Gendler hold radically different views regarding the justificatory force of a thought experiment. However, their disagreement stems in part from the different senses in which they use terms like 'demonstrative' or 'justificatory force'. It is decisive to note that Norton is primarily interested in how the conclusion of a thought experiment is justified (e.g. Galileo's claim that all heavy bodies, regardless of weight, fall at the same rate). However, Gendler's analysis is directed primarily at the process through which we become convinced of the truth of certain background assumptions. Indeed for Gendler, "part of the thought experiment's function" is to bring the reader "to accept certain premises" (1998, p. 408). Paul Humphreys expresses a similar view, in drawing attention to the way in which thought experiments "are directed not only at the principal conclusion but at an exploratory analysis of the conditions on which the thought experiment is predicated" (Humphreys 1993, p. 218). For Gendler, such an exploratory analysis is carried out, not by means of an inductive or deductive inference, but by the contemplation of an imaginary scenario.

On this reading, the justificatory force of a thought experiment rests, not on its logical form, but rather "on its capacity to make available in a theoretical way those tacit practical commitments" which are ultimately necessary to establish the principal conclusion (Gendler 1998, p. 415). Gendler suggests that we often rely on tacit or implicit beliefs about the physical world (about which I will say more later). In doing so, we often come to realize we are committed to certain implicit assumptions, which we were not previously aware of. In this

\footnotetext{
${ }^{2}$ Gendler distinguishes between two ways in which we may take the logical reconstruction of a thought experiment as equivalent to the execution of a thought experiment: (a) the dispensability thesis, according to which "[a]ny good thought experiment can be replaced without loss of demonstrative force, by a non-thought experiment argument", and (ii) the derivativity thesis, according to which "[t]he justificatory force of any good thought experiment can only be explained by the fact that it can be replaced, without loss of demonstrative force, by a non-thought experiment argument. Needless to say, Gendler rejects both theses (1998, p. 401).
} 
sense, a thought experiment brings to light tacit beliefs about the physical world, which would be unavailable to us via standard forms of argument.

But here certain problems arise. To what extent are we really justified in accepting such tacit beliefs? Gendler admits that her account should be understood as "the beginning not the end of an answer to the question", but she nevertheless maintains, "the fact that we have these beliefs gives us prima facie warrant to think that they are true" (Gendler 1998, p. 414). As I will argue below, there are good reasons to be skeptical of this view. I see no reason to suppose that just because we find certain beliefs compelling, or because they are implicit, and to this extent it never occurs to us that they might be false, we are justified in holding them to be true. Norton has raised similar concerns in posing the question: "why should the results of such introspection be credible?" (Norton 2004b, p. 59). Moreover, as I will argue below, such tacit beliefs constitute only one possible source of knowledge invoked in thought experiments.

While Gendler's account suffers from certain difficulties, it nevertheless offers many valuable insights. In focusing my attention on the way in which thought experiments are executed, rather than justified, I maintain we can gain a deeper understanding of how thought experimenters are able to construct arguments by working through imaginary scenarios (and not merely reconstruct them post facto). In this way, it becomes clear why thought experiments enable us to do something that conventional forms of argumentation cannot. One can accept Norton's view the thought experiments can be reconstructed as arguments (the reconstruction thesis), without adopting the view that thought experiments are nothing but arguments (the execution thesis). Conversely, accepting Gendler's view that conducting a thought experiment involves the contemplation of an imaginary scenario does not entail that we commit ourselves to the view that thought experiments employ a form of justification that eludes the standard form of logical analysis.

\section{Gendler's Analysis of Galileo's Thought Experiment}

In order to illustrate Gendler's view, it is useful to review her analysis of Galileo's two-bodies thought experiment. Galileo's thought experiment, which was intended to refute the Aristotelian view that heavy bodies fall faster than light ones is often cited as one of the classic thought experiments in the history of science, and has been the subject of extensive historical and philosophical analysis (Atkinson 2003; Atkinson \& Pejnenburg 2004; J. Brown 1986; Gendler 1998; Koyré 1968; Matthews 2005; Palmerino 2011; Palmieri 2005; Schrenk 
2004). In Galileo's Discourse on the Two New Sciences (1638), written in the form of a dialogue, Simplicio (an Aristotelian) is invited by Salviati to consider what would happen if two stones of different weights were tied together and then dropped. Simplicio initially concludes that if the heavier stone falls faster than the lighter one, "the more rapid one would be partly retarded by the slower", and thus the composite body would fall slower than the heavier of the two stones by itself. Yet, upon further reflection, he concedes that "the two stones when tied together make a stone larger [heavier] than that which moved before", and so it seems reasonable to conclude that the composite body would fall faster than the heavier stone (Galilei [1638] 1968, pp. 62-3). Here Simplicio finds himself trapped in a contradiction - the two bodies tied together would appear to fall faster and slower than the single heavy body. Salviati draws the conclusion that all bodies, regardless of weight, must fall at the same rate (provided they are composed of the same material and fall in the same medium). ${ }^{3}$

In attempting to understand the force of this thought experiment, Gendler provides a logical reconstruction, which I follow closely here. The point of this reconstruction is to show that a simple statement of the premises is insufficient to account for the way the thought experiment works. For a more detailed account, I refer readers to her original 1998 paper.

Gendler begins by presenting a fairly standard logical reconstruction of this thought experiment, by breaking it up into two premises, from which we infer the conclusion.

\section{Natural speed is meditative}

If two bodies fall with different natural speeds $s_{1}$ and $s_{2}$, then the speed of the combined body will lie somewhere between $s_{1}$ and $s_{2}$.

\section{Weight is additive}

This simply states that if two bodies of different weights $\mathrm{w}_{1}$ and $\mathrm{w}_{2}$ are joined together, the total weight of the combined body will be the sum of the two: $\mathrm{w}_{1}+\mathrm{w}_{2}$.

From this Galileo draws the conclusion:

Natural speed is not proportional to weight

\footnotetext{
${ }^{3}$ These conditions in brackets are often neglected in modern presentations of the thought experiment, but we can skip over them for the sake of the reconstruction.
} 
Yet as many authors have pointed out, the initial premises may be challenged. Indeed Gendler suggests a number of ways in which an Aristotelian could respond to Galileo's thought experiment. By considering the scenario more carefully, a committed Aristotelian could call into question the assumptions underlying either or both of the premises. To this end, Gendler provides two alternatives, in the form of two new revised premises:

\section{Natural speed is not physically determined for strapped bodies}

\section{Weight is not physically determined for strapped bodies}

In the first revised premise (3), the Aristotelian may simply reject "the assumption that two stones, when joined together make a single stone and thus have a natural speed which is comparable in principle to that of other stones" (Irvine 1991, p. 161). ${ }^{4}$ In the second revised premise (4), the Aristotelian rejects the assumption that when two bodies are strapped together (both of which have a determinate weight), the combined body possesses a determinate weight. Both are logically available options.

However, as Gendler indicates, there is a third option open to the Aristotelian. By thinking more about this scenario, one might feel that it is preferable to distinguish between bodies that are united (they remain distinct physical entities when strapped together) and bodies that are unified (they become a single entity). If we adopt this distinction, it is possible to replace the initial premises with two alternative premises:

\section{5a. Natural speed and weight are meditative for bodies that are united}

5b. Natural speed and weight are additive for bodies that are unified

Having drawn this distinction, we appear to be confronted with another question: what physical conditions must obtain for two bodies that are strapped together to constitute a truly unified body? Does this depend on how tightly the bodies are strapped together? Are, for example, two bodies loosely tied together with a piece of string unified, or merely united? What about the case in which one body is placed inside the other?

These assumptions also raise another interesting problem. If two falling bodies that were united at some point (perhaps because they loosely bound together) were suddenly to become

\footnotetext{
${ }^{4}$ Miklós Rédei has argued that this "assumption is indeed not self-evident and is in need of empirical testing" (Rédei, 2003, p. 238).
} 
untied, there would be a sudden and discontinuous change in their speed. Such a discontinuity seems contrary to our experience of falling bodies.

One way out of this dilemma, Gendler suggests, is to revise the assumption regarding the clear-cut distinction between united and unified bodies. Instead one could see these as two extreme cases at each end of a continuous spectrum. With this in mind, Gendler suggests the Aristotelian might postulate a "degree of connectedness" $\mathrm{C}(0<\mathrm{C}<1)$, where $\mathrm{C}=1$ corresponds to a completely unified body, while $\mathrm{C}=0$ corresponds to united (but separate) bodies. For all intermediate cases, the value of $\mathrm{C}$ will fall somewhere in between 0 and 1 . With a few simplifying assumptions, ${ }^{5}$ we could write the following formula:

Natural speed of falling bodies $=C\left(s_{1}+s_{2}\right)+(1-C)\left(s_{1}+s_{2}\right) / 2$

Of course, as Gendler admits, taking this option depends on the assumption that the 'degree of connectedness' is a relevant physical property.

Yet, as Gendler suggests, there is something wholly unsatisfactory about all these ways out. They appear to conflict with some deeply held tacit beliefs we have about the nature of bodies. Galileo's original thought experiment, rests on certain assumptions, which were not initially made explicit, but which on closer reflection it appears the Aristotelian shares. These may be stated in the form of two further implicit premises:

\section{All bodies have a determinate natural speed and weight.}

\section{Entification is not a physically determined property.}

Some clarification is necessary here. The first premise (6) states that every body, no matter how we conceive of it, has a particular weight and a natural speed. All bodies possess such physical properties. There is no indeterminate state of affairs. The second premise (7) states that there is no fact-of-the-matter concerning whether we consider a body as a single entity or consisting of many separate bodies. As Gendler explains: "The answer to the question 'how many objects?' does not follow from any physical property we might discover" in the world. It is therefore purely a matter of arbitrary definition whether we regard "a strapped-togetherbody to be a single object, or two objects held together by a strap, or indefinitely many

\footnotetext{
${ }^{5}$ For the sake of simplicity we can assume that (i) the units of speed and weight are the same, and (ii) the natural speed of a unified body is the mean of the natural speeds of each of the two bodies.
} 
objects held together by internal forces" (Gendler, 1998, p. 407). To this extent such considerations should play no part in our analysis of the physics of the situation.

Gendler's reconstruction is intended to bring to light the way in which we are led to form certain assumptions by contemplating an imaginary scenario. Executing a thought experiment is a process of guided contemplation, in which we are confronted with the question: "What would I say/judge/expect were I to encounter circumstances XYZ?” (Gendler, 1998, p. 414). By considering the scenario of the two falling bodies, the Aristotelian gradually becomes aware of "the inadequacy of his conceptual framework for dealing with phenomena which through the contemplation of this imaginary case - he comes to recognize as always having been part of his world" (Gendler 1998, p. 412). In doing so, it becomes apparent that certain "ways out, though logically available, run counter to certain tacit knowledge about the physical world" (Gendler 1998, pp. 404-5). It becomes evident that premises 6 and 7 form an essential part of our thinking about the nature of falling bodies. As Gendler explains:

[B]y thinking about the case in question, we discover what sorts of motions and objects we think are possible in the world. Do we think objects can be strapped together? Yes we do. Do we think objects fall with radical discontinuities in speed? No, we think they do not. Do we think entification is something that is fixed by the world? No, we do not. Do we think weight and natural speed are fixed by the world? Yes, we do. We come to recognize that we have these beliefs by contemplating the imaginary case in question; thinking about the case is what brings us to the realization that we believe what we do (Gendler 1998, p. 414).

In thinking through the scenario, the Aristotelian reluctantly comes to the realization that he holds certain assumptions concerning the nature of falling bodies, which lead to the conclusion that speed is not proportional to weight. It is only by reflecting on the scenario posited in the thought experiment, and by reflecting on our experiences about falling bodies and the nature of bodies in general, that we become aware of certain implicit conceptual commitments. For Gendler, "the Aristotelian comes to have novel justified true beliefs about the empirical world not because he has (whether he knows it or not) followed the path of a recognized argument form, but rather because he has performed an act of introspection that brings to light heretofore unarticulated and (because he lacked a theoretical framework on which to make sense of them) heretofore implausible tacit beliefs" (Gendler 1998, p. 411). This, according to Gendler, brings to light the role of tacit knowledge in the "process of conceptual reorganization" in the execution of a thought experiment (Gendler 2004, pp. 1154). 


\section{The Sources of Knowledge}

The question of 'where the knowledge comes from' when we perform a thought experiment has been the subject of vigorous debate in recent years. James Brown argued, some twenty years ago, that certain thought experiments provide intuitive access to a priori knowledge that "is not based on new empirical evidence nor is it merely logically derived from the old data" (J. Brown 1991, p. 77). According to this view, Galileo's two-bodies thought experiment is an example of what Brown calls a 'platonic' thought experiment. Such thought experiments are able to provide us with some form of intuitive access to certain truths (e.g. all bodies fall at the same rate regardless of weight) via "a kind of perception of the relevant laws of the nature" (J. Brown 1991, ix). This view has drawn sharp criticism from Norton, who contends that that thought experiments provide no such "mysterious route to knowledge of the physical world". They "draw upon what we already know of it", and to this extent, Norton assures us that "empiricism has nothing to fear from thought experiments" (Norton 2004b, p. 45).

The debate between Brown and Norton revolves around the means by which we arrive at the conclusion of a thought experiment. To this extent both the rationalist and the empiricist camps are typically in agreement that the premises "must have been both well known and generally accepted before the [thought] experiment was even conceived" (Kuhn 1977, p. 7). The disagreement is about whether the conclusion can be established via standard forms of deductive or inductive inference. Gendler's account, by contrast, does not focus primarily on how we arrive at the conclusion, but rather the way in which the assumptions or premises gradually come to light in the process of executing a thought experiment. In a certain sense Gendler agrees with both Norton and Brown that thought experiments draw upon what we already know of the world (Norton 2004b). However, she is careful to emphasize that such knowledge is primarily tacit. It is only by contemplating the scenario in question that our beliefs about the world become explicit.

Gendler's emphasis on the tacit knowledge accessed through a thought experiment provides a different way of framing the empiricist viewpoint. In performing a thought experiment, we draw upon knowledge that we have acquired through experience, but this knowledge is not previously available to us in propositional form. This is what, for Gendler, gives thought experiments their unique demonstrative force. Here she appeals to Mach's view that much of our knowledge of the world comes from experiences we have accumulated, but which remain "uncomprehended and unanalysed". As Mach puts it: "In these experiences we possess a treasure-store which is ever close at hand, and of which only the smallest portion is embodied 
in clear articulate thought" (Mach [1883] 1960, p. 36). Gendler makes this aspect of Machian epistemology central to her view:

\begin{abstract}
We have stores of unarticulated knowledge of the world, which are not organized under any theoretical framework. Argument will not give us access to that knowledge because the knowledge is not propositionally available. Framed properly, however, a thought experiment can tap into it, and - much like an ordinary experiment - allow us to make use of information, which was, in some sense, there all along, if only we had known how to systematize it into patterns of which we are able to make sense (Gendler 1998, pp. 414-5).
\end{abstract}

Thought experiments on this account, enable us to acquire "new knowledge of the external world" by a conceptual reorganization of "tacit beliefs ... already held" (Gendler 1998, p. 411). This is reminiscent of Kuhn's earlier account, according to which thought experiments "give the scientist access to information which is simultaneously at hand and yet somehow inaccessible to him" (Kuhn 1977, p. 24). Framing things in this way invariably raises a series of questions familiar to those who have grappled with the paradoxes of tacit knowledge. How we should speak about beliefs about the world that were "there all along", but somehow "inaccessible" or "unavailable"? A deeper exploration of this question would take us far beyond the scope of this paper and into the territory of recent research into cognitive psychology. ${ }^{6}$ Here we may simply note that for Gendler, our access to tacit knowledge serves not only as a source of new beliefs, but also as a source of justification for those beliefs. By accessing our tacit knowledge about the behaviour of falling bodies, we are precluded from accepting certain possibilities that would otherwise be available to us (Gendler, 1998, p. 407). This is what, according to Gendler, gives thought experiments their unique demonstrative force. (Gendler, 1998, p. 415)

Yet as indicated earlier, Gendler's account runs into two crucial problems. The first concerns her claim about justification. Why should we have confidence in our tacit beliefs? As noted earlier, for Gendler, "the fact that we have these beliefs gives us prima facie warrant to think that they are true". (Gendler, 1998, p. 414) Yet this seems an overly optimistic view. Our experiences often turn out to be unreliable, particularly when attempting to judge what would happen in highly idealized scenarios. Consider for example the counterfactual scenarios invoked in Newton's rotating bucket or Stevin's wreath of spheres. While such thought experiments appeal to our everyday experience of spinning objects and bodies sliding down

\footnotetext{
${ }^{6}$ Gendler's position is in many respects reminiscent of one we find in Lakatos' well known work, Proofs and Refutations, in which the protagonists of the dialogue ponder the status of 'hidden assumptions' and 'unconsciously held lemmas' in the analysis of a mathematical proof (Lakatos, 1976).
} 
inclined surfaces, we have obviously never experienced a truly frictionless surface or a world devoid of all other material bodies - and here it is not surprising to find that our experiences may lead us astray.

It is worth noting that Mach himself emphasized the unreliability of these intuitions based on experience. In his analysis of Stevin's famous thought experiment on the wreath of spheres, Mach argued that "the assumption from which Stevin starts, that the endless chain does not move" stems from the conviction that perpetual motion of this kind is impossible. Such a conviction originates in the fact "that we have never experienced anything like a motion of the kind referred to". Yet, as Mach points out, this view "is just as fallible as the distinctly conscious. Its only value is in provinces with which we are very familiar" (Mach [1883] 1960, 43ff). Indeed even in more familiar scenarios, such as the behavior of projectiles inside the cabin of a moving ship, one does not find universal agreement among the thinkers of the $16^{\text {th }}$ and $17^{\text {th }}$ century, about what one would expect to happen. ${ }^{7}$ This seems to run counter to Gendler's view that thought experiments tap into some kind of universal pre-theoretical tacit understanding of the world. Indeed, contrary to what Gendler maintains, thought experiments often force us to revise deeply held tacit beliefs about the world.

The second problem with Gendler's account is that it fails to take into consideration the fact that a great many thought experiments make use of knowledge that is explicitly formulated. While it might well be the case that in considering two falling bodies tied together we draw on tacit beliefs about the nature of falling bodies, this is not a general rule for contemplating imaginary scenarios. There are many thought experiments, particularly from modern physics, that simply do not work this way. Here we should heed Humphreys' warning that "No single thought experiment can possibly represent the variety of uses to which such devices have been put" ((Humphreys, 1993, p. 207). While philosophers frequently pay lip service to this point, in many cases they attempt to draw general conclusions based on the analysis of a

\footnotetext{
${ }^{7}$ Here we can compare the intuitions of Tycho Brahe and Galileo on what would occur aboard a moving ship. In his Episteolarum astronomicarum Tycho claimed: "Indeed certain men think that a missile hurled upwards from a ship, if this would occur inside of the vessel, would fall to the same place if the ship is moving as if it remained motionless. They offer these theories without any reflexion for it actually happens quite differently than they suppose. In fact the swifter the ships advance, the more differences will be discovered. The same thing results from the [supposed] revolution of the earth" (See Overmann, 1975, p. 14). We may contrast this with Galileo's account in the Dialogue on the Two Chief World Systems, which considers almost exactly the same scenario, although arrives at an entirely different conclusion. "So long as the motion is uniform and not fluctuating this way and that", Galileo maintains that objects moving around in the cabin of a ship would undergo no noticeable effects in their motions: "You will discover not the least change in all the effects named, nor could you tell from any of them whether the ship was moving or standing still... the ship's motion is common to all the things contained in it, and to the air also. That is why I said you should be below decks; for if this took place above in the open air, which would not follow the course of the ship, more or less noticeable differences would be seen in some of the effects noted" (Galilei, [1632] 1953, pp. 186-7).
} 
single thought experiment or a particular class of thought experiments. Gendler, here appears to succumb to the tendency to over-generalize.

In certain cases, we are guided by previously acquired theoretical and empirical knowledge, much of which is not only capable of articulation, but has been actually articulated. This is particularly evident when we consider the use of thought experiments in highly abstract theoretical domains, such as quantum mechanics or the theory of relativity, where we rely far more on our working knowledge of theory, rather than any immediate acquaintance with the behavior of sub-atomic particles or objects moving at velocities close to the speed of light. As Gendler points out, "the fact that Norton's primary stable of examples concerns thought experiments in relativity theory (see, e.g., Norton 1991), whereas Brown's concerns thought experiments in early modern science, may explain some of their divergence in analysis". 8 (Gendler, 2004 p. 1161 fn 9) I do not want to claim that we can neatly distinguish between thought experiments that draw on tacit knowledge and those that draw on explicit knowledge. But it is obvious that in many instances our judgments about what would happen in a given imaginary situation are guided by well-established physical theories.

\section{What are Guided Contemplations?}

We are now in a position to examine more carefully what kind of work is performed in executing a thought experiment. Here it is instructive to reflect further on Gendler's treatment of Galileo's thought experiment in section 3. While I agree with Gendler that by contemplating an imaginary scenario we may become aware our previously unarticulated beliefs, I do not share her view that in accessing these tacit beliefs, we are precluded from accepting other possibilities that might otherwise be available to us. In the example discussed above, I would suggest, it is not the case that the Aristotelian is compelled to conclude that speed is meditative, but rather the imaginary scenario forces the Aristotelian (or anyone else for that matter) to take a stance on the question of mediativity. What stance we take will depend on what judgments we form about a range of other relevant questions. Here it is instructive to recall the way in which working through Galileo's thought experiment may forces us to reflect on, and in some cases reassess, our commitment to certain presuppositions such as the 'mediativity of speed', the 'additivity of weight' or 'entification'. While Gendler focuses her analysis on the way in which the contemplation of imaginary scenarios forces us

\footnotetext{
${ }^{8}$ Alisa Bokulich, draws the conclusion that "(for thought experiments in physics) the very description of a thought experiment requires a great many abstract symbolic expressions whose meaning and correspondence with the facts are indicated by theories" (Bokulich 2001, pp. 300-1).
} 
to draw on tacit knowledge, here I wish to emphasize the way in which working through a thought experiment forces us to confront, and even reformulate, certain questions. While in many cases, the answers to such questions may be obvious (e.g. speed is meditative), this is not always so. Attending to this constructive and interrogative dimension of thought experiments is essential to understanding how they work.

The point is easily illustrated by referring to the many different ways in which we may recast Galileo's imaginary scenario involving two falling bodies of different weights tied together. In the Discorsi, Simplicio readily assents to Salviati's proposition that speed is mediative, yet as Gendler and others have shown, in the process of working through the thought experiment we can call into question this assumption. As we have seen, one can simply deny "that two stones, when joined together make a single stone and thus have a natural speed which is comparable in principle to that of other stones" (Irvine 1991, p. 161). This move forces us to redefine the very meaning of a body. In an effort to resolve the paradox presented by Galileo's thought experiment, the Aristotelian could legitimately take the view that "no proper part of a body is itself a body". Indeed, as Roy Sorensen points out, without accepting this view, the Aristotelian who is committed to the view that heavier bodies fall faster than lighter ones would be forced to accept the absurd conclusion "that all proper parts of a body fall slower than the body itself!" (Sorensen 1992, p. 127).

Yet, as Gendler has shown, there are numerous other avenues open to us. We may challenge the formulation of the question rather than proposing an alternative answer. In the two-bodies thought experiment, one need not categorically accept or reject the proposition that speed is meditative, but instead attempt to carefully define the conditions under which the concept of mediativity is applicable. To this end, we may draw a distinction between bodies that are unified (but which remain distinct entities) and bodies that are united (to form a single entity). Yet, as illustrated earlier, this move raises further difficulties. By drawing such a clear-cut distinction, we appear to be committed to the possibility of the radical discontinuity of speed of falling bodies. In order to circumvent such a possibility, we might want to revise our earlier assumption, and postulate a 'degree of connectedness' between objects that are strapped together. Gendler maintains that in working through the imaginary case, it becomes evident that certain possibilities, while logically available, are precluded by the thought experiment itself. However, as I have suggested, this is not necessarily the case. While such possibilities may run counter to our basic intuitions about the world, they may also be provisionally accepted, in working through an imaginary scenario. 
Moreover, it is instructive to note that while most modern commentators have tended to focus on the assumption of the mediativity of speed in their reconstructive analyses, Galileo seems to have been more preoccupied by the hitherto taken-for-granted assumption of the additivity of weight. Paulo Palmeiri has shown that in grappling with the case of two falling bodies in the early 1630s, Galileo was struck by the seemingly unavoidable, but no less astonishing, conclusion that bodies in free fall have no weight. Indeed, this idea finds expression in ensuing discussion of the thought experiment presented in the Discorsi, in which Salviatti explains to Simplicio that in the situation where two bodies fall with the same speed, "it is not true that the smaller stone adds weight to the larger" (Galilei [1638] 1968, p. 67). This view entails a fundamental departure from the Aristotelian concept of weight. Arthur argues that this reading of the thought experiment results in different reconstruction of the premises of the argument from that provided by both Gendler and Norton (Arthur, 1999, p. 226). My aim here is not to argue for a correct reading of the original thought experiment, but to emphasize how contemplating the imaginary scenario in Galileo's thought experiment may lead to different reconstructions involving different assumptions.

Assumptions concerning the medium in which the bodies fall may also come into play. Recently, David Atkinson has argued that in the case of the two bodies falling under the conditions of laminar flow in a highly viscous fluid, each of the falling bodies would approach a terminal velocity proportional to its weight. In this situation we have a physical situation in which speed is meditative. Yet, again this conclusion depends on certain considerations pertaining to the way the bodies are tied or strapped together. Placing one object inside the other would presumably not have the same effect. While Atkinson's suggestion obviously draws on a working knowledge of hydrodynamics not available to Galileo and his contemporaries, the salient point here is that it is by reflecting on the conditions obtaining in the imaginary scenario, not the logical form of an argument, which prompts us to investigate these possibilities. In this situation, an "increased understanding and knowledge is produced by a refinement of the scenario underlying the thought experiment" (Humphreys 1993, p. 218). Indeed Atkinson's reconstruction is perfectly consistent with Galileo's original formulation of the thought experiment, which involves two bodies of the same specific gravity in the same medium.

These considerations, which emerge from Gendler's careful analysis and the work of other philosophers, provide a valuable insight into the workings of a thought experiment. Working through a thought experiment involves a process of responding to, and sometimes even reformulating, questions which arise about the particulars of an imaginary scenario. The premises of the logically reconstructed argument are not stipulations, but rather, they emerge 
out of this interrogative process. This is what makes different reconstructions of the same thought experiment, or what Norton calls thought-experiment/anti-thought-experiment pairs, possible. Michael Bishop has argued that the famous confrontation between Bohr and Einstein at the 1930 Solvay conference over the 'clock-in-the-box' thought experiment is a classic case in which "we have two different arguments [involving different assumptions] but just one thought experiment" (Bishop 1999, p. 540). Norton, on the other hand, steadfastly maintains that in this situation we actually have two different thought experiments corresponding to two different logical arguments (Norton 2004b, pp. 25-26). Yet, on the constructivist account presented here, the problem of how to individuate thought experiments is not an issue that we need take a principled stance on. ${ }^{9}$ Cases such as this, in which contemplation of the same imaginary scenario leads different people, holding different background assumptions, to different premises and conclusions, are quite common in the history of science. ${ }^{10}$ The assumptions we make in contemplating an imaginary scenario will typically depend on our grasp of the relevant body of empirical/theoretical knowledge. As David Gooding puts it: "The efficacy of a TE trades heavily on how well informed its perpetrators are about the world we are invited to explore" (Gooding 1994, p. 1041).

The consideration of these points brings us to consider another important aspect of thought experiments that is often overlooked. The contemplation of an imaginary scenario may bring to light the importance, and even the necessity, of making judgments. By thinking about Galileo's falling bodies scenario, it becomes evident that some judgment about the 'mediativity of speed', or the 'additivity of weight', or what constitutes 'an entity' is relevant for an understanding of the nature of falling bodies. Typically, a thought experiment provides a scenario in which certain background assumptions (such as mediativity of speed and additivity of weight) must be considered together as part of a whole problem-complex. Mere deductive reasoning cannot do this, because it simply takes the set of independent premises of the deductive argument as a given. But as Arthur makes clear, Galileo's thought experiment represents "the phenomenon of fall in such a way that the Aristotelian is reluctantly persuaded of the joint untenability of his premises" (Arthur 1999, p. 222). The imaginary scenario in

\footnotetext{
${ }^{9}$ For Gendler, "How one goes about individuating thought experiments is a question on which I will allow myself to remain neutral: Is Einstein's clock-in-the-box thought experiment (which assumes classical spacetime) the same thought experiment as Bohr's (which assumes relativistic spacetime)? ... Is the thought experiment that I perform when I read Galileo's text the same as the thought experiment Galileo performed when he wrote it? Nothing of what I will go on to say will turn on how these questions - to which it seems difficult to find principled answers - are dealt with" (Gendler 2004, p. $1161 \mathrm{fn} 9)$.

${ }^{10}$ Here we might think of the different elaborations of the EPR thought experiment by Einstein and Bohr, or the versions of Maxwell's demon presented by Smoluchowski, Szillard and Brillouin, or Mach's critical analysis of Newton's spinning bucket thought-experiment.
} 
Galileo's thought experiment provides the epistemic space for considering the set of different (and ultimately revisable) assumptions concerning the nature of falling bodies together.

\section{The Role of Cognitive Skill in Executing a Thought Experiment}

If we turn our attention to a different class of thought experiments, more representative of those found in modern physics, it becomes clear that the execution of a thought experiment invariably relies on various forms of skill or know-how. As Harold Brown has argued:

[T]he accomplishments of science depend on the ability to exercise judgment, an ability that scientists develop as they learn they practice and craft. Scientists develop judgment in the specific fields that they have mastered, and this results in the existence of a set of cognitive skills in the scientific community. These skills are fallible, but their fallibility doe not make them epistemically worthless. Rather our cognitive skills provide genuine grounds for evaluating proposals (H. Brown, 2000, pp. 201-2).

Thought experiments constitute one important example of this kind of cognitive activity. In many cases the ability to execute a thought experiment well frequently depends on the ability to recognize that some piece of previously acquired knowledge is relevant to the situation at hand. A classic example of this can be found in the Einstein-Bohr debate over the 'clock-inthe-box' thought experiment at the 1930 Solvay conference. Einstein's original formulation of the thought experiment was intended to demonstrate that there are physically realistic scenarios that violate the uncertainty relations. Bohr, however, was able to show that this conclusion was valid only under the condition of a classical space-time. By invoking the relativistic energy-time relationship for a gravitational field (ironically taken from Einstein's own general theory of relativity!), Bohr was able to show that in the imaginary scenario posited by Einstein, the uncertainty relations are not violated. While both physicists were obviously familiar with this assumption (indeed Einstein had formulated it!), it was Bohr who saw its relevance for the situation at hand. This illustrates nicely the importance of recognizing the relevance of previously acquired knowledge for executing a thought experiment. ${ }^{11}$ Paul Langevin's famous 'twins paradox', first formulated in 1911, in the context of Einstein's special theory of relativity, provides another historical example of the

${ }^{11}$ One might well question whether Bohr's assumption (of employing a relativistic energy-time relation) was relevant, given that the thought experiment was intended to show the incompleteness of non-relativistic quantum mechanics. It is not at all obvious why certain relativistic assumptions should be relevant, or even necessary, in this context. However, my point here is not to argue for the reevaluation of Bohr's triumph over Einstein. I merely wish to point out that judgments about the relevance of certain assumptions are indispensable for thought experiments. 
way in which physicists formed different judgments about what assumptions were relevant in the contemplation of an imaginary scenario. ${ }^{12}$

It is worth emphasizing that the question of whether a particular assumption is relevant to the execution of a thought experiment is not the same as the question of whether it is strictly applicable. Many thought experiments take place in highly idealized scenarios, in which we are required to neglect certain features of the real world like air resistance, friction, or relativistic or quantum effects. If a thought experiment is to serve its purpose, the idealization should capture the aspects of the world deemed relevant to the phenomenon under consideration. But here again the thought experimenter must exercise judgment. Logical argument can only tell us that in a hypothetical world in which speed is meditative and weight is additive, speed is not proportional to weight. But surely part of the aim of Galileo's thought experiment is to convince us that we $d o$ live in such a world. The idealized world posited in a thought experiment must enable us to draw conclusions about the real world, not merely some hypothetical one, if it is to serve its purpose.

On the basis of the forgoing analysis, we can now address one of the key concerns raised by Norton: "If thought experiments are to be reliable instruments of inquiry then we must have some way of adjudicating between competing thought experiments" (Norton 2004b). I agree with Norton that without a means of adjudication, thought experiments would be useless. However, Norton's conclusion that we are only able to judge whether one thought experiment-argument is better than another one solely on the basis of logical analysis appears problematic. The logical reconstruction of a thought experiment is a necessary, but not sufficient for judging whether it is good or bad. As Gooding puts it: "If the object of reconstruction is to recast narratives into a logical form, then exposing the suppressed premise would be all that is at issue" (Gooding 1994, p. 1035). In the case of thought-experiment/antithought-experiment pairs we may compare the logical structure of the arguments, to identify the hidden assumptions and ensure that the explicitly stated premises are sufficient to justify the conclusion in each case. But once this is done, and we are satisfied that the suppressed premises have been made explicit, the question remains: how do we judge which logical argument is better? In order to answer this question, we would need to make a judgment about which of the two sets of premises are warranted, or to put it another way, which of the assumptions are directly relevant to the phenomenon in question. While this is sometimes

\footnotetext{
${ }^{12}$ In the years from 1911 to 1915 , a number of leading physicists expressed some disagreement about precisely which aspect of relativity theory was relevant for the resolution of the paradox. While it was generally agreed that only one of the twins experienced a change in inertial reference frame, such noted physicists as Max von Laue, Max Born and Albert Einstein offered different explanations of the phenomenon (See Miller 1981, p. 261; Jammer 2006, p. 165).
} 
quite trivial, as the examples above make clear, this is by no means always the case. Indeed I suspect that a detailed historical analysis of the ongoing debates over thought experiments such as Maxwell's demon or the EPR paradox would bring to light both the difficulty and critical importance of exercising this kind of judgment in the contemplation of imaginary scenarios in physics (Ehrenberg, 1967).

The ability to execute a thought experiment well will also frequently depend on another kind of cognitive skill. Even when a certain body of knowledge, or theory, is recognized as relevant to a certain imaginary scenario, it may not be immediately obvious how to apply that knowledge. Many thought experiments involving objects moving at near light speed and undergoing relativistic effects (e.g. the twins paradox, the ladder-barn paradox, the rotating ring paradox) obviously require a certain familiarity with the special (and perhaps also the general) theory of relativity. But even those who have some acquaintance with the formal aspects of the theory often have trouble in working through such thought experiments. Simply knowing the formula for the Lorenz transform for length contraction and time dilation does not automatically mean knowing how to apply it to a particular real or imaginary situation. This practical form of knowledge or skill often proves challenging for students, and demonstrates the way in which a well executed thought experiment does not simply depend on possessing the relevant body of knowledge. As Gooding puts it, "What is needed here is a combination of empirical knowledge and the ability to reason with it" (Gooding 1994, p. $1041)$.

Gooding provides a further example, by asking us to consider "what happens to a very small hole bored through a large metal ring when the latter is heated: does the hole get bigger or smaller?" As Gooding rightly points out, "Besides knowing that heating causes expansion you need to know how to apply this fact (but even then there is room for doubt about the result)" (Gooding 1994, p. 1041). (Indeed I recall my own initial confusion when an undergraduate student first posed this question to me!) In some instances, it may not even be obvious what knowledge is relevant to a particular scenario. In the example presented here, we might ask what else should be taken into account in contemplating the heated ring. One might be tempted to think that the structural properties of the metal, or the exact shape of the ring might be relevant (though in fact they are not). It may not even be apparent whether we have all the relevant information necessary to answer the question. In other words, it may not be immediately clear whether or not the thought experiment is a 'well-defined' problem in the sense of containing all the necessary and sufficient conditions for drawing a conclusion about 
what would happen. ${ }^{13}$ Judgments about what is relevant therefore play an indispensible role in the execution of a thought experiment.

These examples, which require us to apply a physical theory to a particular imaginary scenario, draw attention to one of the important functions of thought experiments. Thought experiments can reveal certain implications of our theories, which often appear surprising. As Alisa Bokulich explains, in considering an imaginary scenario such as the twins paradox, "we begin with a theory or set of assumed laws and then use the thought experiment to uncover certain consequences from these laws that might otherwise remain hidden". In doing so, "the thought experiment makes these consequences explicit" (Bokulich, 2001, p. 301). Indeed in many cases, these consequences may constitute sufficient grounds for reevaluating the theory. To this end, Bokulich claims that thought experiments can and "do play a role in evaluating, accepting, and rejecting theories" (Bokulich, 2001, p. 301).

Here it is instructive to refer to the recent work of Henk de Regt and Denis Dieks (de Regt, 2009; de Regt \& Dieks, 2005). While they do not specifically focus on the role of thought experiments in science, they have argued that the ability to 'see the consequences' of a theory, by applying it to concrete situations, constitutes a particular form of scientific understanding that is crucial for achieving the epistemic aims of science. Here the authors reiterate Gooding's earlier point that having knowledge of a theory is not the same as knowing how to use that theory. Indeed, simply "possessing a theory is not enough: in addition one should be able to use the theory to derive predictions or descriptions of the [real or imaginary] phenomenon" (De Regt \& Dieks, 2005, p. 142). For de Regt, this ability to apply the theory to concrete situations depends a form of pragmatic understanding or knowhow, which involves skill and judgment, and "cannot be reduced to rule-following procedures" (De Regt, 2009, p. 594). The scientist must, as it were, "develop a feeling for the consequences of the theory has in concrete situations" (De Regt \& Dieks, 2005, pp. 142-3). ${ }^{14}$ As I have attempted to show in the examples above, thought experiments constitute one important way in which scientists do this. The execution of a thought experiment is a

\footnotetext{
${ }^{13}$ It is interesting to note that Humphreys argues that thought experiments are not well-defined problems. But this seems too restrictive, and would exclude many cases commonly considered to be good examples of thought experiments, such as the ladder-barn paradox. It seems rather that knowing whether or not a thought experiment is 'well-defined' in the sense of containing all the necessary and sufficient conditions for its resolution is precisely one of the skills of a good thought experimenter (Humphreys 1994).

${ }^{14}$ As de Regt and Dieks make clear: "It is possible to have technical proficiency in manipulating formulas and symbols in the theory, without possessing understanding" and conversely "it is possible to understand how a theory works without being able to do precise calculations with it" (De Regt \& Dieks, 2005, p. 151).
} 
cognitive activity that requires the individual to possess a certain kind of skill and to exercise judgment.

\section{Conclusion: Are Thought Experiments Arguments?}

So let us return to the original question, at the heart of the disagreement between Gendler and Norton. Does a thought experiment consist in the execution of an argument? On the basis of the foregoing analysis, it seems that this very much depends on how we define the word 'execution'. In one sense, a thought experiment can be understood as the execution of an argument, provided we do not take this to mean that it involves nothing more than the mere stipulation of premises, from which we draw a conclusion via induction or deduction. If by the term 'execution', however, we mean the contemplation of the imaginary scenario, which forces us to make judgments, and revise and reformulate our assumptions, then I have no objection to calling this process the execution of an argument. The assumptions we make, both implicit and explicit, can of course serve as premises from which we infer a conclusion, but it would be misleading to think of a thought experiment as entailing nothing more than an exercise in deductive or inductive logic.

The constructivist account presented here draws heavily on Gendler's view, however it departs from her insistence on the primacy of tacit beliefs, and the conception of 'justificatory force' that underpins her account. The process of working through a thought experiment, according to the view defended here, is an interrogative process, and in this sense, it may be understood as one particular way of constructing an argument. But here it is crucial to note that precisely what logical form the argument will take will depend on how we address certain questions in contemplating the scenario, what assumptions we deem relevant to the situation, and how previously acquired knowledge is applied to the situation at hand. This is why different arguments with divergent conclusions (or what Norton calls thoughtexperiment/anti-thought experiment pairs), can often emerge from the contemplation of a single imaginary scenario. A guided contemplation may bring to light certain conceptual ambiguities, which need to be resolved before any definitive conclusion can be drawn.

While I agree with Gendler that thought experiments require some form of tacit knowledge, my account makes no direct appeal to tacitly held beliefs, nor their justificatory force. Of course, previously unarticulated assumptions may emerge in the course of executing a thought experiment, but what is crucial in the process of working through a thought experiment is the need to exercise judgment and having the cognitive skill to do so. In order 
to execute a thought experiment well, scientists must decide whether the problem is well posed, what background assumptions are relevant, what aspects of the scenario can be ignored and how to apply our existing knowledge to the scenario. To this extent, we should the view the execution of a thought experiments as a skillful practice.

This point in no way the diminishes the importance of logical analysis and reconstruction in assessing the validity of a thought experiment. But the focus on the logical structure should not blind us to the fact that judgment, skill and know-how prove to be indispensible in performing thought experiments. Viewed from this perspective, we may see how the ability to reason in imaginary scenarios can play a crucial role in science, not only in attaining conceptual clarity, but also in providing a deeper understanding of scientific theories by making explicit their unforeseen and sometimes surprising consequences. 


\section{References}

Arthur, R. (1999). On thought experiments as a priori science. International Studies in the Philosophy of Science, 13, 215-229.

Atkinson, D. (2003). Experiments and Thought Experiments in Natural Science. In M. C. Galavotti (Ed.), Observation and experiment in the natural and social sciences (pp. 209-225). Dordrecht: Kluwer.

Atkinson, D., \& Pejnenburg, J. (2004). Galileo and prior philosophy. Studies in History and Philosophy of Science, 35, 115-136.

Bishop, M. (1998). An Epistemological Role for Thought Experiments. In N. Shanks (Ed.), Idealization IX: Idealization in contemporary phsyics (pp. 19-33). Amsterdam: Rodopi.

Bishop, M. (1999). Why thought experiments are not arguments. Philosophy of Science, 66, 534-541.

Bokulich, A. (2001). Rethinking thought experiments. Perspectives on Science, 9, 285-307.

Brown, H. I. (1988). Rationality. London; New York: Routledge.

Brown, H. I. (2000). Judgment, Role in Science. In W. H. Newton-Smith (Ed.), A companion to the philosophy of science (pp. 194-202). Oxford: Blackwell.

Brown, J. R. (1986). Thought experiments since the scientific revolution. International Studies in the Philosophy of Science, 1(1), 1-15.

Brown, J. R. (1991). Laboratory of the mind: Thought experiments in the natural sciences. London: Routledge.

De Regt, H. (2009). The Epistemic Value of Understanding. Philosophy of Science, 76, 585597.

De Regt, H., \& Dieks, D. (2005). A Contextual Approach to Scientific Understanding. Synthese, 144, 137-170.

Ehrenberg, W. (1967). Maxwell's Demon, Reprinted from Scientific American (pp. 103-110). San Fransisco: W. H. Freeman \& Co.

Galilei, G. ([1632] 1953). Dialogue on the great world systems (G. de Santillana, Trans.). Chicago: University of Chicago Press.

Galilei, G. ([1638] 1968). Dialogues concerning two new sciences (H. Crew \& A. d. Salvio, Trans.). Evanston, Chicago: Northwestern University Press. 
Gendler, T. S. (1998). Galileo and the indispensability of scientific thought experiments. British Journal for the Philosophy of Science, 49, 397-424.

Gendler, T. S. (2000). Thought experiment: On the powers and limits of imaginary cases. New York: Garland Press (now Routledge).

Gendler, T. S. (2002). Thought experiment. Encyclopedia of Cognitive Science. New York; London: Nature; Routledge.

Gendler, T. S. (2004). Thought experiments rethought - and reperceived. Philosophy of Science, 71, 1152-1164.

Gooding, D. (1993). What is experimental about thought experiments? PSA 1992, vol. 2, 280290.

Gooding, D. (1994). Imaginary science. British Journal for the Philosophy of Science, 45, 1029-1045.

Humphreys, P. (1993). Seven theses on thought experiments. In J. Earman (Ed.), Philosophical Problems of the Internal and External World: essays on the philosophy of Adolf Grünbaum (pp. 205-227 ). Pittsburgh: University of Pittsburgh Press.

Irvine, A. D. (1991). Thought Experiments in Scientific Reasoning. In T. Horowitz \& G. Massey (Eds.), Thought Experiments in science and philosophy (pp. 149-165). Savage, MD Rowman and Littlefield.

Jammer, M. (2006). Concepts of simultaneity: From antiquity to Einstein and beyond. Baltimore: The Johns Hopkins University Press.

Koyré, A. (1968). Galileo's Treatise De motu gravium: The tse and abuse of imaginary experiment Metaphysics and measurement: Essays in the scientific revolution (pp. 44-88). Cambridge: Harvard University Press.

Kuhn, T. (1977). A function for thought experiments. The essential tension (pp. 240-265). Chicago: University of Chicago Press.

Lakatos, I. (1976). Proofs and refutations: the logic of mathematical discovery. Cambridge; New York: Cambridge University Press.

Mach, E. ([1883] 1960). The science of mechanics (J. McCormack, Trans.). LaSalle Illinois: Open Court

Matthews, M. (2005). Idealisation and Galileo's penulum discoveries: Historical, philosophical and pedagogical considerations. In M. R. Matthews, C. F. Gauld \& A. Stinner (Eds.), The pendulum: Scientific, historical and educational perspectives (pp. 209-235). Dordrecht: Springer. 
Miller, A. I. (1981). Albert Einstein's special theory of relativity. Emergence (1905) and early interpretation (1905-1911). Reading, Massachusetts: Addison-Wesley.

Norton, J. (1996). Are thought experiments just what you always thought? Canadian Journal of Philosophy, 26(3), 333-366.

Norton, J. (2004a). On thought experiments: Is there more to the argument? Proceedings of the 2002 Biennial Meeting of the Philosophy of Science Association, Philosophy of Science, 71, 1139-1151.

Norton, J. (2004b). Why thought experiments do not transcend empiricism Contemporary debates in the philosophy of science (pp. 44-66). Oxford: Blackwell.

Overmann, R. J. (1975). Theories of gravity in the seventeenth century. Unpublished $\mathrm{PhD}$, Indiana University, Indiana.

Palmerino, C. R. (2011). Galileo's use of medieval thought experiments. In K. Lerodiakonou \& S. Roux (Eds.), Thought experiments in methodological and historical contexts (pp. 101-125). Leiden: Brill.

Palmieri, P. (2005). Spuntar lo scoglio più duro': Did Galileo ever think the most beautiful thought experiment in the history of science? Studies in History and Philosophy of Science. Part A, 36(2), 223-240.

Rédei, M. (2003). Thinking about thought experiments in physics: Comment on 'experiments and thought experiments in natural science'. In M. C. Galavotti (Ed.), Observation and experiment in the natural and social sciences (pp. 237-241). Dordrecht: Kluwer.

Schrenk, M. A. (2004). Galileo vs. Aristotle on free falling dodies. Logical Annalysis and History of Philosophy, 7(1), 1-11.

Sorensen, R. (1992). Thought experiments. Oxford: Oxford University Press. 